\title{
Vent'anni di scritture migranti: una nuova letteratura, una nuova lingua
}

\section{Serge Vanvolsem $†$}

\section{(2) OpenEdition}

1 Journals

\section{Edizione digitale}

URL: https://journals.openedition.org/cher/11253

DOI: 10.4000/cher.11253

ISSN: 2803-5992

\section{Editore}

Presses universitaires de Strasbourg

\section{Edizione cartacea}

Data di pubblicazione: 30 juin 2013

Paginazione: 103-111

ISBN: 978-2-35410-054-4

ISSN: 1968-035X

Notizia bibliografica digitale

Serge Vanvolsem †, «Vent'anni di scritture migranti: una nuova letteratura, una nuova lingua», reCHERches [Online], 10 | 2013, online dal 08 février 2022, consultato il 10 février 2022. URL: http:// journals.openedition.org/cher/11253 ; DOI: https://doi.org/10.4000/cher.11253

\section{(c) (i) (2)(2)}

Ce(tte) œuvre est mise à disposition selon les termes de la Licence Creative Commons Attribution Pas d'Utilisation Commerciale - Partage dans les Mêmes Conditions 4.0 International. 


\title{
Vent'anni di scritture migranti: una nuova letteratura, una nuova lingua
}

\author{
Serge VanVOLSEM $\dagger$ \\ Katholieke Universiteit Leuven ${ }^{1}$
}

\begin{abstract}
$\mathrm{N}$ ella seconda metà del secolo scorso l'Italia, quasi senza accorgersene, almeno in un primo tempo, si è trasformata da paese di emigrazione in paese di immigrazione. La prima fase, ben nota e descritta, ha portato via dall'Italia fra ventisei e ventisette milioni di cittadini, che, secondo Armando Gnisci, dato il periodo storico delle prime partenze, non hanno fatto in tempo "a diventare italiani, ma diventarono americani e australiani" ${ }^{2}$. A parte il fatto che, credo, si possa diventare italiani anche fuori d'Italia - basta guardare i milioni di tifosi che, attaccati allo schermo, seguono le partite degli azzurri o i Gran Premi di Formula Uno - l'etichetta certamente non vale per le centinaia di migliaia di emigrati che si sono stabiliti in Europa. Venuti per la maggior parte dopo la prima e soprattutto dopo la seconda guerra mondiale, non appartengono più direttamente al periodo formativo
\end{abstract}

1 Questo testo è postumo ed è l'ultimo lavoro di Serge Vanvolsem, che la famiglia ci ha gentilmente concesso di pubblicare. Si tratta della relazione destinata al convegno di Strasburgo «Ecrire ailleurs. Littérature et migration en Italie (1990-2010)». La decisione di farlo apparire in questa sede vuole essere innanzitutto un omaggio alla memoria di un grande studioso, fra i primi ad essersi interessato alla letteratura migrante italiana, ma anche un atto di rispetto della volontà dell'autore che di questo saggio parla in una nota al suo articolo Dagli elefanti a nonno Dio. Il rinnovo del codice linguistico italiano con le scritture migranti in Leggere il testo e il mondo. Vent'anni di scritture della migrazione in Italia, a cura di F. Pezzarossa e I. Rossini, Bologna, Clueb, 2011, nota 5, p. 3. Nella nota 11 abbiamo accorpato alcuni appunti che non potevamo includere nel testo. [n.d.c.]

2 Cfr. A. Gnisci e N. Moll, Breviario per conoscere la letteratura italiana della migrazione, Roma, Provincia di Roma, Assessorato alle politiche culturali, 2010, p. 6. 
del nuovo stato. Con queste ultime ondate prende praticamente fine ciò che è stato chiamato il grande esodo storico.

A partire dagli anni Settanta partenze e ritorni sono in equilibrio, l'Italia cessa di essere un paese di emigrazione, ma nello stesso periodo è cominciata ad essere un paese di immigrazione. Il paese per questo, come aveva poi già dimostrato il commercio medioevale, si trovava idealmente situato sia nei corridoi est-ovest, sia sulla rotta sud-nord, e l'ingresso in uno dei paesi dell'Unione Europea facilitava ovviamente la circolazione all'interno dell'Unione. Così anche oggi; e molti si sono trovati bene in Italia o vi sono comunque rimasti per necessità. I primi anni i flussi non sono stati massicci, e passavano, come dicevo all'inizio, quasi inosservati. Ecco come l'albanese Vladimir Koçirai commenta, un po' ironicamente, il silenzio intorno all'arrivo degli albanesi in Puglia, dopo un primo interessamento da parte della stampa.

La traversata è diventata talmente abituale che non se ne parla più, non ci si fa più caso. E questo fatto, a dir la verità, a noi non ci fa tanto piacere; ci sentiamo abbandonati dalla stampa, non abbiamo più il nostro spazio nei telegiornali. Così la gente, gli albanesi, ma soprattutto gli italiani, non si sentono più informati. Cari signori italiani, io come pilota, come portatore di uno di questi mezzi, sento il dovere di tranquillizzarvi: non preoccupatevi! Cari signori, la traversata si effettua regolarmente. L'Albania non vi lascerà mai soli ${ }^{3}$.

Ricordo che stampa e televisione erano soprattutto sorprese di vedere come molti di questi albanesi fossero in grado di esprimersi in italiano, un italiano imparato a forza di ascoltare e guardare clandestinamente la radio e la televisione italiana, per molto tempo uniche finestre sul mondo occidentale libero. Nel frattempo, perché non voglio fare qui la storia di quest'immigrazione, questi primi immigrati sono diventati più di 4,5 milioni: ottocentomila romeni, quattrocentoquarantamila albanesi, quattrocentomila marocchini, centosettantamila cinesi, centocinquantamila ucraini, per nominare solo le comunità maggiori ${ }^{4}$. Rappresentano il 7,2\% della popolazione, il che vuol dire che in Italia un abitante su quattordici è uno straniero. Come sempre, non solo in Italia, l'arrivo di forestieri, specie se diventa massiccio, viene considerato come elemento di disturbo e non sono mancati e non mancano tuttora episodi di intolleranza e di razzismo.

3 V. Koçirai, Il gommista di Valona, in AA. VV., Il doppio sguardo. Culture allo specchio, Roma, Adnkronos Libri, 2002, p. 59.

4 Fonte il Dossier Statistico 2009 sull'immigrazione. 
Le parole extracomunitario, per indicare chi è immigrato da paesi poveri, e vu cumprà, per designare i venditori ambulanti soprattutto di origine africana, entrano nel vocabolario negli anni Ottanta ed hanno all'inizio certamente un'accezione spregiativa ${ }^{5}$.

Lo scopo primario di tutti i migranti, checché sia stato il motivo della migrazione, è senza dubbio quello di rifarsi una vita fuori del paese d'origine. Vale anche qui l'adagio primum vivere deinde philosophari: gli immigrati devono sistemarsi e trovare un lavoro per poter vivere. Non si va negli Stati Uniti o in Australia per scrivere versi, non si lascia il Senegal per pubblicare racconti o romanzi a Milano; quindi per veder nascere una vita culturale occorre aspettare che la comunità si assesti, che riceva le possibilità di espressione artistica $^{6}$. Non voglio discutere qui se quest'espressione artistica sia un vero dono della natura o solo lo sfogo di una carica emotiva traboccante. Specie per autori di un solo testo, si può trattare di sublimazione di una troppo grande emozione: migrare non è come andare in vacanza all'estero, è un'esperienza molto profonda e traumatizzante; migrare può far scrivere, anche se questo non significa produrre capolavori letterari. Buona parte delle scritture migranti certamente non supera il livello di testimonianze delle proprie condizioni di vita e lavoro in una società più o meno accogliente. Un'altra osservazione che va fatta a proposito dello sfasamento dell'espressione artistica di una comunità rispetto al momento dell'arrivo nei nuovi territori, è che questo non esclude affatto esperienze individuali diverse. Il poeta albanese Gëzim Hajdari, ad esempio, ha abbandonato la sua patria per motivi politici e come tutti ha conosciuto

5 Sia il Grande dizionario italiano dell'uso (Gradit) di De Mauro, sia il Dizionario della lingua italiana di Sabatini e Coletti (Disc) danno come data di prima attestazione di extracomunitario il 1980 e di vu cumpra' il 1986. Il dossier didattico Materiali di lavoro, preparato da Patrizia Restiotto e Alessandro Micheletti, e che costituisce la seconda parte dell'edizione del 2002 di La promessa di Hamadi contiene un glossario che fa risalire vu cumpra' già al 1925: «l'espressione compare la prima volta nel 1925, nella poesia di Raffaele Viviani O' tripolino, riferita al caso di un napoletano trasferitosi in Libia come venditore ambulante», p. 157.

6 Questo periodo serve anche per il tirocinio linguistico e spiega perché i primi volumi vengono scritti a quattro mani. In un intervento pubblicato su Internet nel 2005 Saidou Moussa Ba parla esplicitamente di questa necessità di collaborazione con un italiano per corrispondere all'urgenza di scrivere: «Certamente in quegli anni parecchi di noi hanno sentito l'esigenza di avviare una comunicazione con la società ospitante attraverso la scrittura, aiutati da italiani. Forse si poteva raggiungere, prima di pronunciarci, una padronanza linguistica più sicura, ma l'esigenza della necessità di farci conoscere, di interrogare e interrogarci è stata superiore ad ogni prudenza di attesa» (cfr. l'Archivo di El Gibli, Anno 2, $\mathrm{n}^{\circ}$ 10, dicembre 2050, supplemento sul sito http://www.el-ghibli.provincia. bologna.it $/$ index.php?id $=2 \&$ issue $=02 \_10 \&$ sezione $=3 \&$ testo $\left.=0 \ldots\right)$. 
anche lui dei problemi per, diciamo, "campare", ma essendo già poeta in patria non ha avuto bisogno di vent'anni per rimettersi a scrivere versi, traducendoli in un primo tempo dall'albanese, ma poi procedendo anche in modo opposto.

Le scritture migranti hanno ormai più di un secolo: ben centoventicinque anni separano gli scritti di oggi dal famoso Peppino (1882) di Luigi Donato Ventura ${ }^{7}$. Con i vent'anni del titolo della relazione non voglio quindi prendere in considerazione né l'intera produzione letteraria della migrazione, né quanto scritto in italiano dagli italiani all'estero, come definito da Jean-Jacques Marchand quando nel 1990 convocò a Losanna gli specialisti per un convegno sugli scrittori di lingua italiana nel mondo ${ }^{8}$. Intendo soffermarmi solo su quanto scrivono o hanno scritti gli immigrati non italiani approdati, legalmente o no, in Italia in questi ultimi due decenni. Questa nuova immigrazione inizia, come detto, negli anni '70, ma comincia a manifestarsi letterariamente, con il solito sfasamento, solo vent'anni dopo negli anni '90. In questa prospettiva il congresso di Losanna, anche se non riguarda direttamente gli scrittori discussi qui, è interessante perché ha certamente favorito la presa di coscienza in Italia. Proprio nel 1990 escono in Italia, e non sempre presso editori minori, i primi libri della scrittura migrante: Immigrato di Mario Fortunato e Salah Methnani (Roma, Theoria; ora Milano, Bompiani) e Io, venditore di elefanti. Una vita per forza fra Dakar, Parigi e Milano di Pap Khouma e Oreste Pivetta (Milano, Garzanti; ora anche Baldini \& Castoldi); seguono poi nel 1991 Chiamatemi Ali di Mohamed Bouchane, curato da Carla Di Girolamo e Daniele Miccione (Milano, Leonardo) e La promessa di Hamadi di Alessandro Micheletti e Saidou Moussa Ba (Novara, De Agostini). Come si vede, i primi testi sono stati scritti a quattro o più mani, ma ben presto questi autori pubblicheranno anche da soli. Le edizioni milanesi dell'Arco, che nel 2004 hanno festeggiato i primi dieci anni di attività, hanno messo in circolazione oltre cento titoli, mentre la banca dati Basili, del Dipartimento di italianistica e spettacolo

7 Luigi Donato Ventura (1845-1912) scrisse la novella del lustrascarpe nel 1882, in italiano, in francese ed in inglese, probabilmente per uso didattico, ma il testo uscì a stampa solo nel 1885, in francese. La versione inglese fu pubblicata un anno dopo. Nel 2007 Martino Marazzi ne ha curato una edizione trilingue: L.D. Ventura, Peppino il lustrascarpe, Milano, Angeli, 2007.

8 Per gli atti si veda J.-J. Marchand (a cura di), La letteratura dell'emigrazione. Gli scrittori di lingua italiana nel mondo, Torino, Edizioni della Fondazione G. Agnelli, 1991. 
della "Sapienza" di Roma, nella rubrica "scrittori e opere letterarie" contiene ben 1320 dati. $^{9}$

Com'erano nuovi per l'Italia i flussi migratori degli anni '70, così era completamente nuova per il mondo letterario l'espressione artistica scritta degli anni '90. I primi scritti degli immigrati erano per la maggior parte autobiografici: Pap Khouma, ad esempio, racconta il lungo viaggio dal Senegal all'Italia, la vita di un immigrato e i diversi atteggiamenti degli italiani con cui viene a contatto, ma siamo a mille miglia dal Viaggio in Italia di Goethe (Italienische Reise, 1817) o da Roma, Napoli e Firenze (Rome, Naples et Florence, 1817) e dalle Passeggiate romane (Promenades dans Rome, 1829) di Stendhal. In tutti i casi si tratta di esperienze vissute, ma Goethe praticamente ha ignorato la popolazione locale. Gli immigrati che scrivono, invece, stanno nella popolazione e parlano della classe più emarginata: appunto gli extracomunitari, i vu cumpra', i clandestini. Hanno sperimentato sulla propria pelle le difficoltà e le umiliazioni per trovare un lavoro come venditore ambulante o come raccoglitore di pomodori. Anche chi è arrivato con tanto di diploma ${ }^{10}$ deve rifarsi una vita e spesso, per così dire, deporre il suo status di intellettuale per ricominciare da zero. Rispetto a Goethe o Stendhal è una tematica fondamentalmente diversa. Il rinnovamento del codice letterario, poi, non deve essere necessariamente spettacolare, basta introdurre una prospettiva nuova. L'importanza della memoria, ad esempio, è certamente una caratteristica importante, sia nelle (auto)biografie, sia anche nei racconti. Ma la memoria letteraria a cui siamo più abituati, è quella della tradizione proustiana, con i suoi accenti sulle sinestesie che vi stanno alla base, sul mondo metaforico, e sul carattere evocativo. É quasi tutta rivolta al passato che si vuole ricordare o rendere presente. La memoria dei racconti delle scritture migranti, invece, senza voler negare tutti questi aspetti tradizionali, ha in primo luogo una prospettiva futura, vuole trasmettere alle generazioni future. Mi ricordo, a questo proposito, un vecchio minatore del Limburgo, deluso che i figli, tutti occupati ad ancorare la loro vita al Belgio, fossero stufi di sentire per

9 Ma la Banca dati, creata da Armando Gnisci e consultabile all'indirizzo www. disp. let.uniroma1.it/basili, non distingue fra pubblicazioni autonome e poesie o racconti pubblicati in riviste o volumi collettivi.

10 In un racconto che ha partecipato al primo concorso di Eks\&Tra, l'edizione 1994-1995, lo zairese Paul Bakolo Ngoi scrive: «Nella compagnia degli africani del Casertano, Kalombo non era l'unico a possedere un titolo di studio. Molti di coloro che venivano dalla Francia e dalla Svizzera erano studenti universitari, senza borsa di studio, che venivano a sbarcare il lunario con la raccolta dei pomodori» (Vista da Kalo, in A. Ramberti e R. Sangiorgi (a cura di), Le voci dell'arcobaleno, Santarcangelo di Romagna, Fara Editore, 1995, p. 59). 
l'ennesima volta la sua storia e le sue nostalgie; ma voleva pubblicare le sue memorie perché - mi diceva - «voglio che sappiano anche i nipoti».

Il rinnovamento del codice letterario si esprime anche nel mondo allegorico e metaforico. Chi viene da culture completamente diverse non può non avere un immaginario particolare che arricchisce e rinnova la visuale tradizionale. Già da noi la nozione di campagna differisce profondamente per chi vive in città in un appartamento, e per chi viene dalla campagna, come libertà e liberazione assumono valori diversi per chi ha conosciuto la seconda guerra mondiale e per le generazioni nate dopo. Anche con parole semplici e tutte italiane si possono creare immagini forti. "Dormo la mia prima notte italiana» scrive Khouma, dopo una serie di peripezie, «notte italiana» e non «in Italia»; e chi non si ricorda i versi pluricitati, anche da me, di Hajdari:

Piove sempre
in questo
paese
forse perché sono
straniero $^{11}$.

di cui scrivono anche i nostri scrittori della migrazione ${ }^{12}$.

Ha dovuto imparare, di notte, ai tempi del suo soggiorno a Cesenatico, quando di giorno vendeva appunto elefantini, maschere e altri oggetti di artigianato africano (sì o no contraffatti dai tanti piccoli laboratori clandestini in Campania) ${ }^{13}$.

11 Pap, p. 28/Eks\&Tra/ tre generazioni e più lingue.

12 È un settore tutto da analizzare; temi: italiani visti da altri + africani (cfr inleiding Neyla); oralità frasi brevi; che patisce Alì, cioè Mohamed Bouchane stesso, come clandestino; sbarcare il lunario; dialetto/Boccaccio http://www.dizionario.org/d/index.php?pageurl=c assamadia\&searchfor=cassalin\&searching=true

Bibliografia: Caritas/Migrantes, Immigrazione Dossier Statistico 2009. XIX Rapporto sull'immigrazione, Roma, Centro Studi e Ricerche Idos, 2009 [cfr. anche www. dossierimmigrazione.it/schede/pres2009.htm]; L. D. Ventura, Peppino il lustrascarpe, edizione trilingue a cura di M. Marazzi, Milano, Angeli, 2007 (ristampa 2008);

M. Fortunato e S. Methnani, Immigrato, Milano, Bompiani, 2006; Goethe in Italia (1786-1788); Stendhal trascorre sette anni a Milano (dal 1814 al '22); nel 1829 le Promenades dans Rome. http://www.meltingpot.org/articolo4511.html; http://www. eksetra.net/stampa/stampa.php?id=14.

13 Io, venditore di elefanti. Una vita per forza fra Dakar, Parigi e Milano, Garzanti, 1990 [nel testo cito dall'edizione 1997]. 
In un caso analogo in Belgio, il romanzo Ritorno a Salicia ${ }^{14}$, del 1993, raccontato da Carmelo Sità (1911-1), e direi "messo in musica" per la pubblicazione da Franco Caporossi, i due autori, che figurano tutti e due sulla copertina, hanno poi litigato rivendicando ognuno la paternità del testo.

Come straniero Pap non può non partire da un immaginario culturale completamente diverso. Sono ormai quarant'anni che faccio l'italianista, ma questi studi non hanno fatto di me un italiano; sono fiammingo ed il mio orizzonte culturale è pieno dei cieli della pittura fiamminga, sereni, nuvolosi, coperti, tempestosi... Ho visto tanti quadri italiani con cieli splendidi dipinti dagli artisti più famosi; cieli realistici con nubi, uccelli etc., o cieli immaginari con mostri, cieli astratti, evocativi, ma erano sempre cieli italiani, diversi dai nostri.

14 F. Caporossi e C. Sità, Ritorno a Salicia. Storia di un emigrante calabrese Zì Carmelo Sità, Reggio Calabria, Laruffa Editore, 1993. Il caso di Ritorno a Salicia è, però, notevolmente più complesso, perché del romanzo esistono anche dei quaderni dattiloscritti (in tutto 150 pagine). I quaderni non sono né firmati né datati, ma sembrano di pugno di Sità (i nomi propri e la toponomastica sono spesso fonetici: Collar per il ministro Léo Collard, Laugni per Longwy, Sciarleroà per Charleroi, Fattlei Managa per Fayt-lez-Manage..., forme che Caporossi, addetto al Consolato italiano non poteva non conoscere) e risalgono probabilmente al 1968 (il testo comincia con «Il 14 luglio 1986 ho compiuto 76 anni [...] $\mathrm{mi}$ sono reso conto che devo scrivere le peripezie e le tristezze della mia vita). Sità mi ha assicurato che i quaderni risalivano a piccoli quaderni manoscritti pieni di appunti che $\mathrm{mi}$ avrebbe procurato perché rimasti probabilmente a casa sua in Calabria; purtroppo la sua morte glielo ha impedito. Il testo del volume a stampa (318 pagine, quindi più del doppio) è molto diverso, ampliato fortemente e con una struttura narrativa notevolmente rifatta (esplicitare i dialoghi spesso resi in modo indiretto nei quaderni, ed altro). In una nota manoscritta nel mio esemplare del romanzo Caporossi dice, a questo proposito che: «Il caso Carmelo Sità è solo il personaggio del romanzo biografico, ma a scriverlo sono stato io. Gli ho concesso di inserire il suo nome al mio come autore per puro rispetto ed affetto nei suoi confronti»; nella Prefazione, datata novembre 1992 precisa che; «Ho scritto questo romanzo biografico seguendo le rievocazioni e alcuni appunti dello stesso protagonista». 